EPJ Web of Conferences 88, 01016 (2015)

DOI: $10.1051 /$ epjconf/ 20158801016

(C) Owned by the authors, published by EDP Sciences - SIF, 2015

\title{
Clustering in dilute matter and equation of state
}

\author{
S. TYPEL
}

GSI Helmholtzzentrum für Schwerionenforschung, Darmstadt, Germany

\begin{abstract}
The chemical composition and thermodynamic properties of strongly interacting matter are strongly affected by cluster correlations at subsaturation densities. A generalized relativistic density functional approach, which includes clusters as explicit degrees of freedom with medium dependent properties, is able to describe the formation and dissolution of clusters. Adapting the density functional for the equation of state to nuclear structure calculations, the occurence of $\alpha$-particle clusters on the surface of heavy nuclei is predicted, which reduces the neutron skin thickness of neutron-rich heavy nuclei and affects the correlation between the neutron skin thicknes and the density dependence of the symmetry energy.
\end{abstract}

\section{Introduction}

Correlations are an essential feature in strongly interacting many-body systems whose properties are encoded in the equation of state (EoS). The study of heavy-ion collisions in laboratory experiments or of the surface of heavy nuclei can help to deduce information on the EoS of dilute matter with densities smaller than nuclear saturation density $n_{\text {sat }} \approx 0.15 \mathrm{fm}^{-3}$. Such conditions are also found in astrophysical objects such as core-collapse supernovae or the crust of neutron stars. However, one has to distinguish between nuclear matter, where only strongly interacting particles are considered but not the electromagnetic interaction, and stellar matter with both hadrons 
and leptons as constituents and the additional condition of charge neutrality. The former system shows a 'non-congruent' liquid-gas phase transition at not too high temperatures $T$ in isospin asymmetric matter with baryon number densities $n_{b}$ below $n_{\text {sat }}[1,2]$. The latter system exhibits the formation of 'pasta' structures and the gas-solid phase transition to a crystal lattice of ions immersed in a sea of electrons.

\section{Generalized relativistic density functional}

In order to incorporate the cluster formation and dissolution in an efficient way in a theoretical model, a generalized relativistic density functional (gRDF) approach [3] was developed by extending well-known relativistic mean-field models with density dependent meson-nucleon couplings [4] using a thermodynamic consistent grand canonical formulation. Baryons (nucleon, hyperons, ... ), nuclei and leptons (in case of stellar matter) are considered as explicit degrees of freedom. They are treated as quasiparticles with medium dependent properties encoded in scalar and vector self-energies. The model parameters are found from a fit to properties of finite nuclei [3]. The effective interaction shows very reasonable nuclear matter parameters, which are compatible with most of current constraints.

Light and heavy clusters are considered as constituents with binding energies taken from the 2012 Atomic Mass Evaluation [5] when available or, if not, from predictions of the DZ10 model [6]. Nucleon-nucleon scattering correlations are included in order to reproduce the correct low-density limit, which is given by the model-independent virial equation of state [7]. The scalar self-energies of composite particles contain medium-dependent mass shifts that originate mainly from the action of the Pauli exclusion principle. This leads to a reduction of the binding energies and the Mott effect, i.e. the dissolution of clusters, can be described. A simple parametrization of the mass shifts is used for the light clusters in the gRDF calculations [3]. Similar mass shifts can be deduced for heavy nuclei in the medium from fully selfconsistent spherical Wigner-Seitz cell calculations in an extended ThomasFermi approximation [8]. An additional Coulomb mass shift appears in stellar matter since the almost uniform electron background screens the Coulomb field. This effect is more pronounced for heavy nuclei.

A strong variation of the chemical composition of matter is caused by the changing in-medium properties of composite particles, see, e.g., the right panel of fig. 10 in ref. [8]. Only two-body correlations are relevant at low densities and the composition is dominated by nucleons and deuteron-like cor- 
relations. With increasing density at given temperature and isospin asymmetry, first the abundance of deuterons increases and then three- and fournucleon cluster contributions become more prominent. The clusters dissolve just below the nuclear saturation density and nucleonic matter remains at higher densities. Effects of cluster formation have to be implemented in EoS models in order to provide a reliable input to astrophysical simulations.

\section{$3 \quad$ Symmetry energy and neutron skins}

The isospin dependence of the energy in nuclear matter can be quantified by the symmetry energy, which measures the difference of the energy per baryon in pure neutron matter and symmetric nuclear matter. The density dependence of the symmetry energy, which is often expressed with the help of the slope parameter $L$, or that of the neutron matter EoS is tightly correlated with the neutron skin thickness $r_{\text {skin }}$ of heavy nuclei, i.e. the difference between the neutron and proton root-mean-square radii [9-11], see, e.g., fig. 2 in ref. [12], which also summarizes many experimental attempts to measure these quantities. The observed $r_{\text {skin }} \leftrightarrow L$ correlation is based solely on relativistic and non-relativistic mean-field calculations of nuclei. They do not take into account clustering effects which might affect the thickness of the neutron skin.

The finite-temperature gRDF approach in spherical Wigner-Seitz cells using an extended relativistic Thomas-Fermi approximation for the description of heavy-nuclei in the medium, see ref. [8], can be adapted to the case of zero temperature and nuclei in vacuum, for details see ref. [13]. The occurrence of $\alpha$ particles on the surface of heavy nuclei is observed in these self-consistent calculations.

For the chain of tin nuclei, see figs. $1-4$ in ref. [13], a systematic reduction of the neutron skin thickness is found with the largest effect for nuclei midway between neutron-proton symmetric nuclei and the most neutronrich tin nuclei. The actual amount of $\alpha$ particles reduces with increasing neutron excess.

The dependence of the neutron skin thickness on the symmetry energy at saturation and the slope parameter $L$ was studied by varying the strength and density dependence of the coupling for the isovector $\rho$ meson. Fixing the quantity $L$ to particular values, several new parameter sets were determined by fits to properties of finite nuclei as for the original DD2 parametrization, see table 1 of refer. [13]. With the formation of $\alpha$ particles on the surface of ${ }^{208} \mathrm{~Pb}$ a similar correlation between $\Delta r_{\text {skin }}$ and $L$ is observed as is standard 
mean-field calculation, see fig. 5 in ref. [13], but the neutron skin thickness is reduced by approx. $0.02 \mathrm{fm}$ or about $10 \%$. This effect should be taken into account in determining $L$ from experimental measurements of the neutron skin thickness, at least as a systematic uncertainty.

The prediction of $\alpha$-cluster correlations on the surface of heavy nuclei can be tested experimentally with quasifree $(\mathrm{p}, \mathrm{p} \alpha)$ reactions. Such investigations are planned for the future at RCNP Osaka [14].

\section{Acknowledgements}

This work was supported by the Helmholtz Association (HGF) through the Nuclear Astrophysics Virtual Institute (VH-VI-417). The author gratefully acknowledges discussions and collaboration with S. Antić, T. Aumann, D. Blaschke, T. Klähn, G. Poghosyan, G. Röpke, T. Uesaka, M. Voskresenskaya and H. H. Wolter.

\section{References}

[1] Müller H. and Serot B.D., Phys. Rev. C 52 (1995) 2072.

[2] Typel S. et al., Eur. Phys. J. A 50 (2014) 17.

[3] Typel S. et al., Phys. Rev. C 81 (2010) 015803.

[4] Typel S. and Wolter H. H., Nucl. Phys. A 656 (1999) 331.

[5] Wang M. et al., Chinese Physics C 36 (2012) 1603.

[6] Duflo J. P. and Zuker A. P., Phys. Rev. C 52 (1995) R23.

[7] Horowitz C. J. and Schwenk A., Nucl. Phys. A 776 (2006) 55.

[8] Typel S., AIP Conf. Proc. 1520 (2013) 68.

[9] Brown B. A., Phys. Rev. Lett. 85 (2000) 5296.

[10] Typel S. and Brown B. A., Phys. Rev. C 64 (2001) 027302.

[11] Furnstahl R., Nucl. Phys. A 706 (2002) 85.

[12] Viñas X. et al., Eur. Phys. J. A 50 (2014) 27.

[13] Typel S., Phys. Rev. C 89 (2014) 064321.

[14] Aumann T. and Uesaka T., private communication, 2014. 